\title{
Theranostic Approach for Cancer Treatment: Multifunctional Gold Nanorods for Optical Imaging and Photothermal Therapy
}

\author{
Oshra Betzer, Rinat Ankri, Menachem Motiei, and Rachela Popovtzer \\ Faculty of Engineering and Institute of Nanotechnology \& Advanced Materials, Bar-Ilan University, 52900 Ramat Gan, Israel \\ Correspondence should be addressed to Rachela Popovtzer; rachela.popovtzer@biu.ac.il
}

Received 20 August 2015; Accepted 25 October 2015

Academic Editor: Kimberly Hamad-Schifferli

Copyright ( 2015 Oshra Betzer et al. This is an open access article distributed under the Creative Commons Attribution License, which permits unrestricted use, distribution, and reproduction in any medium, provided the original work is properly cited.

\begin{abstract}
A critical problem in the treatment of cancer is the inability to identify microsized tumors and treat them without normal tissue destruction. While surgical excision of tumors is highly effective, residual micrometastases and remaining positive margins are the main cause of recurrence. In this study, we propose a theranostic approach for the detection and therapy of head and neck cancer (HNC). We developed a plasmonic-based nanoplatform for combined, ultrasensitive in vivo spectroscopic detection and targeted therapy of HNC. This detection method involves near-infrared (NIR) spectroscopy of gold nanorods (GNRs) that selectively target and attach to squamous cell carcinoma HNC cells, through an immune complex. Diagnosis is based on a spectral shift analysis, which is generated by interparticle-plasmon-resonance patterns of the specifically targeted GNRs. Additionally, the ability to design the GNRs to strongly absorb light in the NIR region enables efficient irradiation of these GNRs, for selective photothermal therapy (PTT) of the cancer cells. We expect this targeted, noninvasive, and nonionizing spectroscopic detection method to provide a highly sensitive and simple diagnostic tool for micrometastasis. In addition, the concomitant development of targeted PTT, based on specific cancer markers, may pave the way for tailoring effective therapy for patients, toward an era of personalized medicine.
\end{abstract}

\section{Introduction}

Head and neck cancer (HNC) is the fifth most common cancer worldwide [1]. A critical problem in cancer therapy is the inability to detect microsized tumors and treat these tumors without destruction of normal tissue. While surgical excision of tumors is highly effective, residual micrometastases and remaining positive margins are the main cause of recurrence.

Over the past decade, targeted contrast agents have become mainstay imaging tools, enabling selective detection and imaging of biological events and processes at cellular and molecular levels. The potential use of gold nanoparticles in near-infrared (NIR) imaging has been shown using a variety of noble metal nanostructures [2], including gold nanoshells [3-8], gold nanorods (GNRs) $[9,10]$, and gold nanocages $[11,12]$. Due to the specific attachment of gold nanoparticles to damaged sites, they can be applied as contrast agents for various optical molecular imaging techniques for cancer detection and therapy, for example, X-ray [13], computed tomography (CT) [14, 15], surface-enhanced Raman scattering $[16,17]$, photoacoustic tomography (PAT) [18], and diffusion reflection measurements $[19,20]$. Each differs in terms of its sensitivity, resolution, complexity, time of data acquisition, and cost but all methods are based on the specific attachment of the gold nanoparticles to the damaged site. However, studies demonstrate that nonspecific binding of gold nanoparticles always occurs, yielding large background signals $[21,22]$.

In the present study, we propose a novel NIR imaging and therapeutic method for HNC, utilizing immunotargeted contrast agents, in which the background signal caused by nontargeted probes does not affect or contribute to the monitored signal. This method is based on monitoring the interparticle resonance (IPR) spectral shift of GNRs when irradiated with NIR light. IPR is a phenomenon in which the resonant wavelength peak of two interacting particles is generally red-shifted (shifted to lower photon energy) from that of a single particle, due to near-field coupling 
$[23,24]$. Moreover, the magnitude of the shift decays approximately exponentially with increasing particle spacing and becomes negligible when the gap between the two particles exceeds $\sim 2.5$ times the particle diameter or short-axis length [23]. Herein, we utilized the near-field interparticle plasmon resonance interactions between adjacent GNRs. When the targeted GNRs are in close proximity (e.g., attached to the cell's surface antigens), they form a unique cluster pattern. In this cluster, the electromagnetic fields of adjacent nanoparticles overlap, resulting in a significant red shift of the resonance wavelength peak from that of individual particles. Nontargeted gold nanoparticles, which randomly spread in the sample, do cause a signal shift and therefore do not modify the monitored signal. Using our method, the redshifted resonant wavelength peaks will be obtained only when the GNRs are attached to the specific antigen pattern of HNC cells. If there are no cells of interest in the sample, the measured resonant wavelength peak will be the same as that of single GNRs. We chose to use GNRs due to recent studies showing that the absorption cross section of nanorods is larger than that of nanocages and more than twice the NIR plasmon resonance of nanoshells [25].

Squamous cell carcinoma (SCC) represents more than $90 \%$ of all head and neck cancers and is characterized by a significant overexpression of epidermal growth factor receptor (EGFR) antigen $[26,27]$. Herein, we suggest detection of SCC with high signal-to-noise ratio, following intravenous administration of targeted GNRs coated with antiEGFR monoclonal antibody. Our method distinguished between specific (targeted) and nonspecific binding of the functionalized nanoparticles in vitro and in vivo, eliminated the background signals originating from nontargeted GNPs, and eliminated background signals created by stray light. Furthermore, we harnessed the sensitivity of cells to small increases in temperature (hyperthermia) for anticancer treatment $[28,29]$, using our system for efficient photothermal therapy (PTT) of the targeted SCC-HNC cells. As the GNRs strongly absorb light in the NIR region, rapid heating of their surrounding environment occurs. This selectively destroys the attached cancer cells, without exposing noncancerous cells to elevated temperatures.

\section{Materials and Methods}

2.1. Nanorods Fabrication and Targeting. GNRs were synthesized using the seed mediated growth method [30]. Their size, shape, and uniformity were characterized using transmission electron microscopy (TEM). GNRs were synthesized with average dimensions of $55 \times 25 \mathrm{~nm}$, resulting in an aspect ratio $(\equiv R)$ of 2.2 and an average effective radius $\left(r_{\text {eff }}\right)$ of $18.6 \mathrm{~nm}$ and absorption peak in $670 \mathrm{~nm}$. The GNRs, termed $\mathrm{GNR}_{670}$, were coated with either heterofunctional PEG covalently conjugated to an anti-EGFR monoclonal antibody or IgG as negative control (antibody and antigen obtained from Erbitux, Merck KGaA, Germany) [31].

2.2. In Vivo Tumor Bearing Mice Model. In vivo measurements were evaluated using mice bearing human $\mathrm{HNC}$ derived from an A-431 SCC cell line. A-431 cells $\left(2 \times 10^{6}\right)$ were injected subcutaneously into the back flank area of 1011 -week-old nude mice. When the tumor reached the size of $5-7 \mathrm{~mm}$ in diameter, the mice received $120 \mu \mathrm{L}$ of immunotargeted GNRs $(48 \mathrm{mg} / \mathrm{mL})$ by tail vein injection.

All in vivo measurements were performed under appropriate anesthesia: the mice barrier-controlled facility was under the strict care of the veterinarian in charge of the Institutional Animal Care and Use Committee (IACUC). The mice were inspected daily by the veterinarian, who handles the appropriate tests and treatment protocols, as required. All research protocols were followed closely by the veterinarian. All major procedures were performed in the surgical facilities using general anesthesia and standard, aseptic surgical techniques.

2.3. Hyperspectral Imaging System. Dark field images of SCC cells and reflectance measurements of cancerous and noncancerous tissues were captured using the hyperspectral imaging system (Nuance, CRi, MA, USA). Xenon and Halogen (UN2-PSE100, Nikon, Japan) illuminations were used for dark field and specular reflectance experiments, respectively, along with $40 \mathrm{x}$ objective ( $0.75 \mathrm{NA}$ ) and a 32-bit ultrasensitive CCD camera detector (N-MSI-EX) for imaging in RGB mode. Microscopy was then performed with a Nikon $80 \mathrm{i}$ Microscope (Nikon Instruments, Inc.). Images were acquired using the Nuance software version 2.1. For each spectral analysis, three regions of interest (ROIs) from the tumor picture were chosen, each presenting the largest region in focus.

2.4. Photothermal Therapy Measurements. PTT measurements were performed on tumor bearing mice $20 \mathrm{~h}$ after intravenous administration of GNRs, to maximize intratumoral particle accumulation prior to NIR exposure. A femtosecond Ti:Sapphire laser (Mai-Tai-Series, Spectral physics, Santa Clara, CA, USA) was employed at a wavelength of $705 \mathrm{~nm}$, due to wavelength overlap with the absorption band of the GNRs $(650-750 \mathrm{~nm})$. The pulse width was 200 femtoseconds each, with a repetition rate of $80 \mathrm{MHz}$. The peak power was $75 \mathrm{~kW}$ and the average power was $0.5 \mathrm{~W}$. The laser beam was directed at the mice tumor site for 5 minutes of illumination.

\section{Results}

3.1. Gold Nanorod Fabrication and Targeting. GNRs were synthesized and characterized. Size, shape, and uniformity of the synthesized $\mathrm{GNR}_{670}$ characterized using TEM showed an average dimension of $55 \times 25 \mathrm{~nm}$, resulting in an aspect ratio $(\equiv R)$ of 2.2 , an average effective radius $\left(r_{\text {eff }}\right)$ of $18.6 \mathrm{~nm}$, and an absorption peak in $670 \mathrm{~nm}$ (Figure 1(a)). GNRs were designed with a resonance wavelength of $670 \mathrm{~nm}$, tuned to the "tissue transparency" window of 600-1000 nm, to increase the laser penetration depth. The GNRs were coated with either heterofunctional PEG covalently conjugated to an antiEGFR monoclonal antibody, specific to head and neck SCC tumors and nontoxic at therapeutic concentrations [31] and which served for cell targeting, or IgG, whose related antigen 


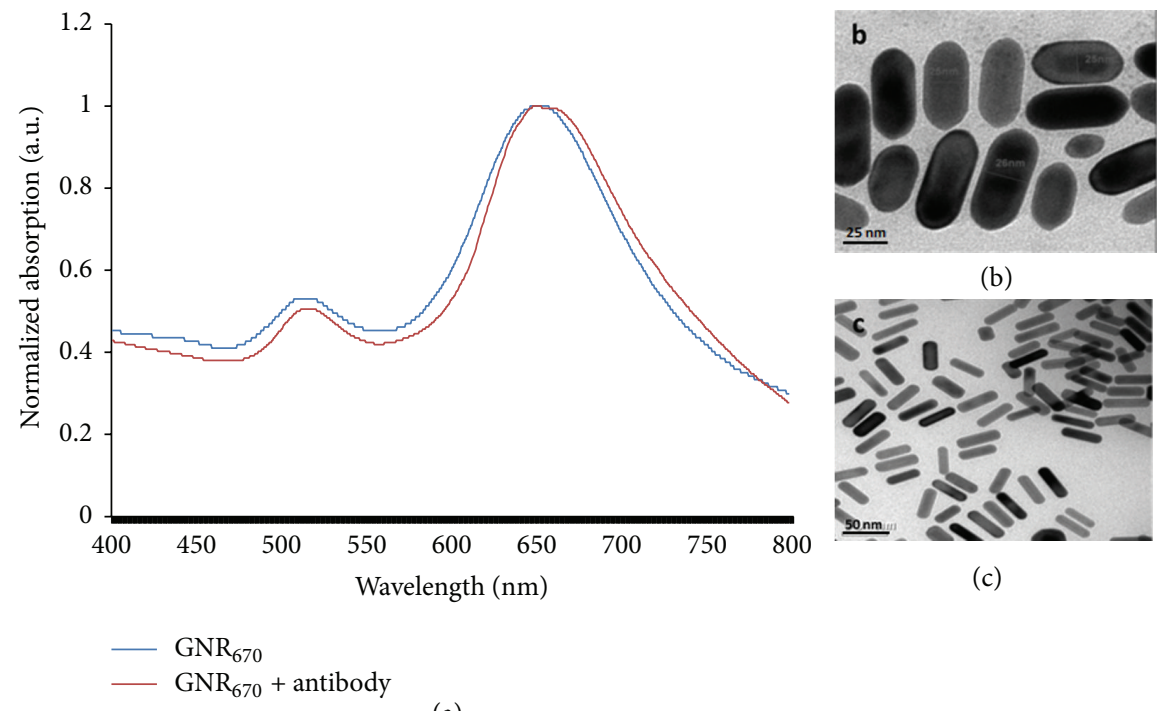

(a)

Figure 1: (a) Optical properties of GNRs. Ultraviolet-visible spectroscopy (UV-Vis) of GNR 670 (blue) and GNR 670 coated with antibody (red). (b-c) Transmission electron microscopy (TEM) images of GNR $_{670}$ (scale bars $25 \mathrm{~nm}$ and $50 \mathrm{~nm}$ ).

was not expressed on SCC cells, which served as a negative control.

\subsection{Light Scattering from Targeted and Nontargeted SCC-HNC} Cells. Using dark field microscope imaging, we found that anti-EGFR coated GNRs bound specifically, and at high concentrations, to the surface of SCC-HNC cells (Figure 2(a)). In contrast, SCC-HNC exposed to nonmatching IgG coated GNRs yielded only a negligible amount of scattered light (Figure 2(b)), resulting from nonspecific binding.

3.3. In Vitro IPR Spectral Red Shift Measurements. The spectral shift of the SCC cell lines was tested using an ultravioletvisible spectroscopy (UV-Vis) spectrophotometer. We found a significant spectral red shift for SCC-HNC cells targeted with anti-EGFR coated GNRs as compared to the control samples, which included SCC-HNC targeted with IgG and noncancerous fibroblasts cells targeted with anti-EGFR$\mathrm{GNR}_{670}$ (Figure 3). The features of specifically targeted GNRs dominate (peak at $700 \mathrm{~nm}$ ) and the shift from the control sample is about $30 \mathrm{~nm}$. These results display a very clear example of the spectral shift and demonstrate the IPR effect of specific immunotargeting of cancer cells.

3.4. In Vivo IPR Spectral Red Shift Measurements. Tumorinducing A-431 cells were subcutaneously injected to mice, followed by intravenous injection of anti-EGFR coated $\mathrm{GNR}_{670}(48 \mathrm{mg} / \mathrm{mL})$ when the tumor reached a diameter of 5-7 mm. Sixteen hours later, two sites in the tumor bearing mice were irradiated, namely, the tumor site and a noncancerous tissue (muscle). The reflected light intensity was collected using hyperspectral imaging microscopy (Figure 4(a)). Figure 4(b) shows UV-Vis bright field and dark field images of the cancerous and noncancerous tissues. Although the normalized GNR reflectance spectra indicated presence of GNRs in both cancerous and noncancerous tissue, reflectance intensity was approximately three times higher for the cancerous tissue (Figure 4(c)). A spectral red shift, resulting in an additional small peak at $720 \mathrm{~nm}$, was also found for the cancerous tissue. These results indicate accumulation of the immunoconjugated GNRs at the tumor site, rather than random dispersion as seen in the noncancerous tissue.

3.5. In Vivo Photothermal Therapy of Targeted SCC Head and Neck Cancer Cells. To examine the efficacy of PTT, tumor bearing mice received intravenous administration of GNRs followed by NIR irradiation (20 h after injection, extracorporeal; $705 \mathrm{~nm}, 5 \mathrm{~mm}$ beam diameter; $5 \mathrm{~min}$ at $0.5 \mathrm{~W}$ ) or control treatments of either NIR irradiation (using the same parameters) without GNR administration or GNR administration without irradiation. Tumor site diameter was measured before laser NIR irradiation and on days 3, 6, 11, and 14 after irradiation. Figure 5 shows that, following GNR injection, NIR illumination of the tumor caused a dramatic decrease in average tumor growth (by $>80 \%$, relative to control tumors) at day 14 . It is notable that mice body weight was not affected by PTT treatment and remained at an average of $21 \pm 2$ gr through the 14 -day experiment.

\section{Discussion}

In the current study, we show that immunoconjugated GNRs can target SCC-HNC cells with high specificity, distinguishing between cancerous and noncancerous tissues. In addition, the combination of this detection method with PTT can effectively decrease tumor size, with minimal side effects. 


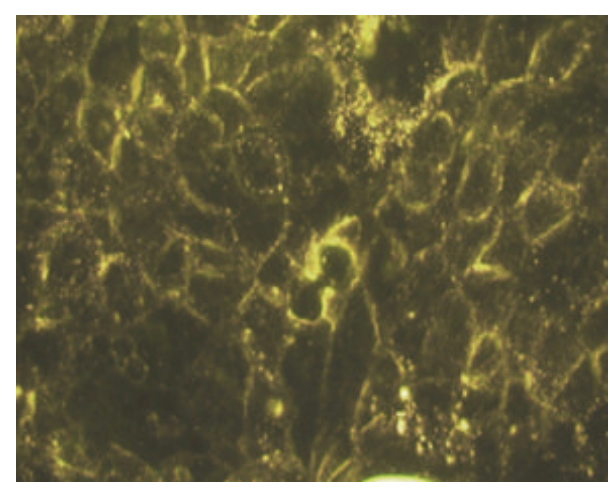

(a)

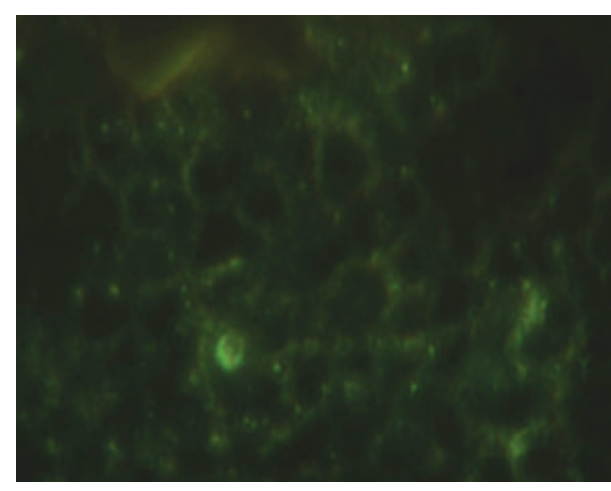

(b)

FIGURE 2: Dark field microscopy images of SCC-HNC cells uploaded with GNRs. (a) Cells specifically targeted with anti-EGFR antibody coated GNRs. (b) Cells incubated with nonmatching IgG coated GNRs.

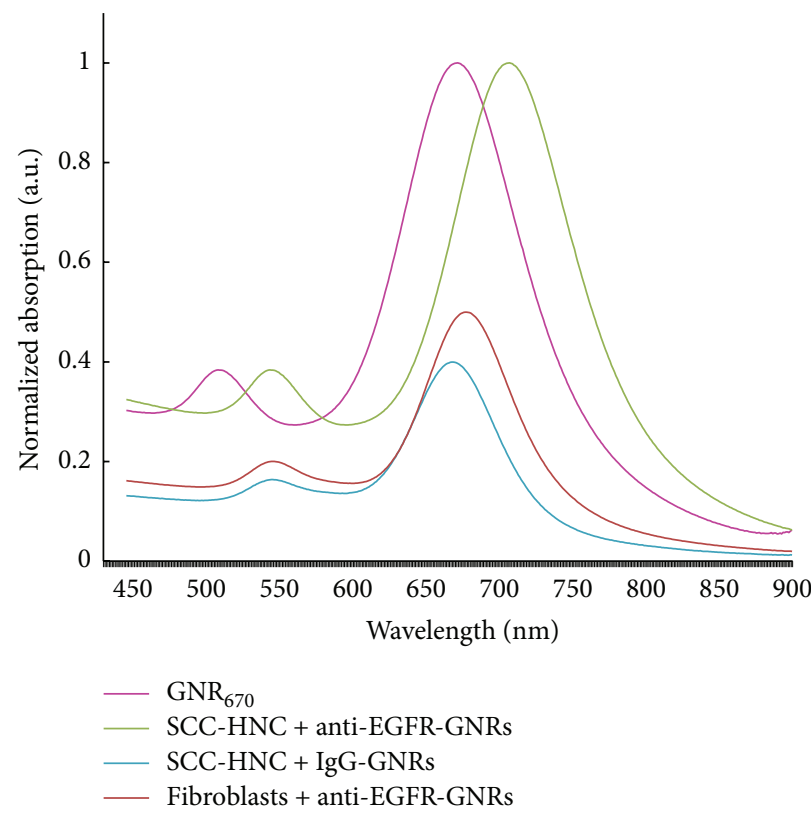

FIGURE 3: In vitro IPR effect on cancer cells: UV-Vis of SCC-HNC targeted with anti-EGFR coated GNRs (green line) and, as control, antibody coated GNRs (either anti-EGFR or IgG) (pink line), SCC-HNC targeted with IgG coated GNRs (blue line), and noncancerous fibroblasts cells targeted with anti-EGFR coated GNRs (red line).

In general, the output spectra from NIR illumination may result from a single gold nanoparticle, which can be found either when there are no SCC cancer cells, and nanoparticles are randomly spread, or due to nonspecific interactions between the nanoparticles and different biological molecules. Alternatively, the spectra may derive from clusters of closely attached gold nanoparticles, which predominately originate from targeted enveloping of the SCC cell surface via antibody-antigen interactions, and are constrained by the receptors' specific biological pattern. Our results, showing that SCC cancer cells targeted by anti-EGFR antibody coated GNRs demonstrate high reflectance intensity, suggest a definite discrimination between the two distinct spatial patterns and thus enable clear-cut discrimination between cancerous and noncancerous tissue.

We used the ability of GNRs to absorb enough light to kill the targeted SCC cells, without affecting nontargeted cells. Thus, the high density of anti-EGFR antibody binding to the overexpressed antigen on SCC-HNC cells induced a significant decrease in tumor growth $(>80 \%)$.

One of the major diagnosis challenges in $\mathrm{HNC}$ is reliable detection of involved lymph nodes, as their status is one of the most important prognosis predictors, and is also pivotal for appropriate treatment. However, assessment of lymph nodes based on the currently available imaging techniques is limited in sensitivity and specificity and fails to distinguish between 


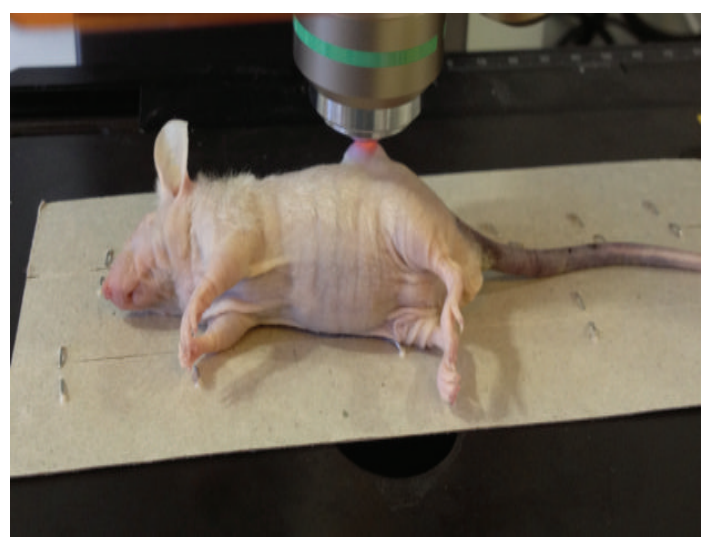

(a)
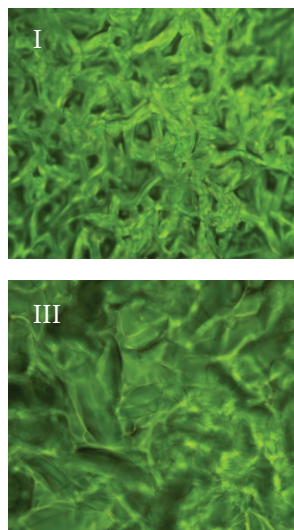

(b)
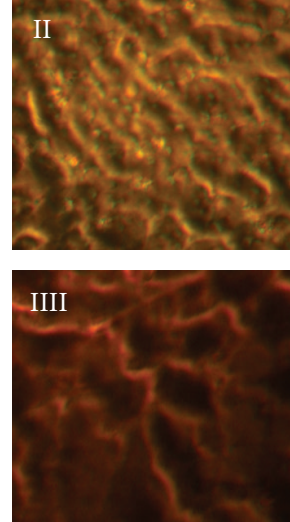

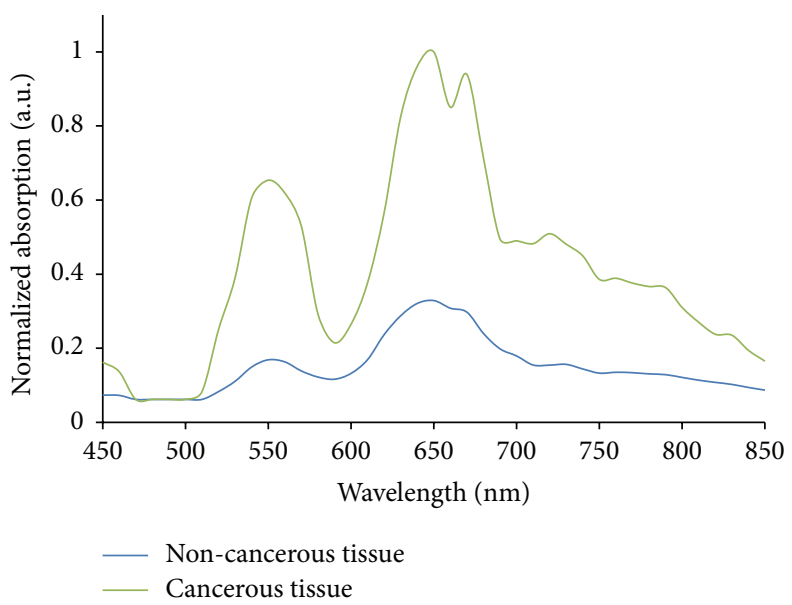

(c)

FIGURE 4: In vivo reflectance intensity and spectral red shift of specifically targeted GNRs. (a) Hyperspectral imaging microscopy setup. (b) Ultraviolet-visible spectroscopy (UV-Vis) bright field and dark field images of the cancerous (I-II) and noncancerous (III-IV) tissues. (c) UV-Vis spectroscopy presenting normalized reflectance spectra for the cancerous and noncancerous tissues, demonstrating the reflectance intensity and spectral red shift of the specifically targeted GNRs.

nonneoplastic and malignant processes. These limitations lead to the routine performance of prophylactic procedures, such as extensive neck dissection and radiation $[1,32]$. HNC and its lymph node metastasis are located adjacent to the skin, where the NIR light can easily penetrate. Therefore, our technique is highly applicable for skin adjacent tumors such as HNC. The successful implementation of our IPR-based detection and therapeutic technique can promote further investigation on the correlation between the amount of cancer cells and markers and spectral parameters such as IPR width and height.

\section{Conclusions}

In the current paper, we propose the development of dual-mode targeted plasmonic nanoprobes as a theranostic approach for the detection and therapy of skin adjacent tumors. We showed, both in vitro and in vivo, that immunetargeted GNRs can target SCC-HNC cancer cells with high specificity and facilitate the differentiation between cancerous and noncancerous tissues. In addition, this specific detection method can be easily and successfully combined with PTT, showing minimal side effects. This targeted, noninvasive, and nonionizing spectroscopic detection method can provide a highly sensitive, simple, and inexpensive diagnostic tool for micrometastasis. In addition, the concomitant development of targeted PTT, based on specific cancer markers, may pave the way for tailoring effective therapy for individual patients, toward the era of personalized medicine.

\section{Conflict of Interests}

The authors declare that there is no conflict of interests regarding the publication of this paper.

\section{References}

[1] D. Chin, G. M. Boyle, S. Porceddu, D. R. Theile, P. G. Parsons, and W. B. Coman, "Head and neck cancer: past, present and future," Expert Review of Anticancer Therapy, vol. 6, no. 7, pp. 1111-1118, 2006. 


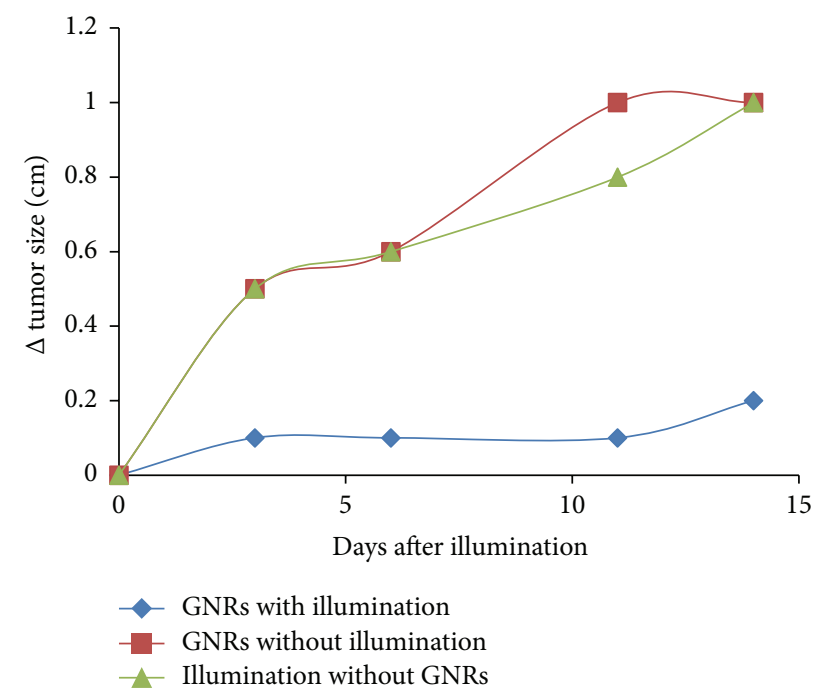

FIgURE 5: Changes in tumor growth after PTT treatment. Tumor diameter measurements on days $3,6,11$, and 14 in mice that received either targeted GNRs with NIR, NIR only, or GNRs only.

[2] L. A. Austin, M. A. MacKey, E. C. Dreaden, and M. A. El-Sayed, "The optical, photothermal, and facile surface chemical properties of gold and silver nanoparticles in biodiagnostics, therapy, and drug delivery," Archives of Toxicology, vol. 88, no. 7, pp. 1391-1417, 2014.

[3] N. Halas, "Playing with plasmons: tuning the optical resonant properties of metallic nanoshells," MRS Bulletin, vol. 30, no. 5, pp. 362-367, 2005.

[4] C. Loo, A. Lowery, N. Halas, J. West, and R. Drezek, "Immunotargeted nanoshells for integrated cancer imaging and therapy," Nano Letters, vol. 5, no. 4, pp. 709-711, 2005.

[5] N. Halas, "The remarkable optical properties of gold nanoshells," Gold Bulletin, vol. 37, no. 1-2, p. 137, 2004.

[6] N. Halas, "Nanoshells as multimodality nanoscale sensors," Abstracts of Papers of the American Chemical Society, vol. 227, p. U107, 2004.

[7] V. Weber, A. Feis, C. Gellini, R. Pilot, P. R. Salvi, and R. Signorini, "Far- and near-field properties of gold nanoshells studied by photoacoustic and surface-enhanced Raman spectroscopies," Physical Chemistry Chemical Physics, vol. 17, no. 33, pp. 21190-21197, 2015.

[8] M. Gordel, J. Olesiak-Banska, R. Kolkowski, K. Matczyszyn, M. Buckle, and M. Samoc, "Shell-thickness-dependent nonlinear optical properties of colloidal gold nanoshells," Journal of Materials Chemistry C, vol. 2, no. 35, pp. 7239-7246, 2014.

[9] X. Huang, I. H. El-Sayed, W. Qian, and M. A. El-Sayed, "Cancer cells assemble and align gold nanorods conjugated to antibodies to produce highly enhanced, sharp, and polarized surface Raman spectra: a potential cancer diagnostic marker," Nano Letters, vol. 7, no. 6, pp. 1591-1597, 2007.

[10] P.-C. Wu, W.-S. Wang, Y.-T. Huang et al., "Porous iron oxide based nanorods developed as delivery nanocapsules," Chemistry - A European Journal, vol. 13, no. 14, pp. 3878-3885, 2007.

[11] M. A. Mahmoud and M. A. El-Sayed, "Comparative study of the assemblies and the resulting plasmon fields of LangmuirBlodgett assembled monolayers of silver nanocubes and gold nanocages," The Journal of Physical Chemistry C, vol. 112, no. 37, pp. 14618-14625, 2008.

[12] S. E. Skrabalak, J. Chen, L. Au, and X. Lu, "Gold nanocages for biomedical applications,” NIH Public Access, vol. 19, pp. 31773184, 2008.

[13] J. F. Hainfeld, D. N. Slatkin, T. M. Focella, and H. M. Smilowitz, "Gold nanoparticles: a new X-ray contrast agent," British Journal of Radiology, vol. 79, no. 939, pp. 248-253, 2006.

[14] T. Reuveni, M. Motiei, Z. Romman, A. Popovtzer, and R. Popovtzer, "Targeted gold nanoparticles enable molecular CT imaging of cancer: an in vivo study, International Journal of Nanomedicine, vol. 6, pp. 2859-2864, 2011.

[15] R. Meir, K. Shamalov, O. Betzer et al., "Nanomedicine for cancer immunotherapy: tracking cancer-specific T-cells in vivo with gold nanoparticles and CT imaging," ACS Nano, vol. 9, no. 6, pp. 6363-6372, 2015.

[16] X. Qian, X.-H. Peng, D. O. Ansari et al., "In vivo tumor targeting and spectroscopic detection with surface-enhanced Raman nanoparticle tags," Nature Biotechnology, vol. 26, no. 1, pp. 8390, 2008.

[17] H. Karabeber, R. Huang, P. Iacono et al., "Guiding brain tumor resection using surface-enhanced Raman scattering nanoparticles and a hand-held Raman scanner," ACS Nano, vol. 8, no. 10, pp. 9755-9766, 2014.

[18] Q. Zhang, N. Iwakuma, P. Sharma et al., "Gold nanoparticles as a contrast agent for in vivo tumor imaging with photoacoustic tomography," Nanotechnology, vol. 20, article 39, Article ID 395102, 2009.

[19] R. Ankri, V. Peretz, M. Motiei, R. Popovtzer, and D. Fixler, "A new method for cancer detection based on diffusion reflection measurements of targeted gold nanorods," International Journal of Nanomedicine, vol. 7, pp. 449-455, 2012.

[20] R. Ankri, D. Leshem-Lev, D. Fixler et al., "Gold nanorods as absorption contrast agents for the noninvasive detection of arterial vascular disorders based on diffusion reflection measurements," Nano Letters, vol. 14, no. 5, pp. 2681-2687, 2014.

[21] H. Chen, L. Wang, J. Yeh et al., "Reducing non-specific binding and uptake of nanoparticles and improving cell targeting with an antifouling PEO-b-P $\gamma$ MPS copolymer coating," Biomaterials, vol. 31, no. 20, pp. 5397-5407, 2010.

[22] L. Brannon-Peppas and J. O. Blanchette, "Nanoparticle and targeted systems for cancer therapy," Advanced Drug Delivery Reviews, vol. 64, pp. 206-212, 2012.

[23] K.-H. Su, Q.-H. Wei, X. Zhang, J. J. Mock, D. R. Smith, and S. Schultz, "Interparticle coupling effects on plasmon resonances of nanogold particles," Nano Letters, vol. 3, no. 8, pp. 1087-1090, 2003.

[24] S. Roh, T. Chung, and B. Lee, "Overview of the characteristics of micro- and nano-structured surface plasmon resonance sensors," Sensors, vol. 11, no. 2, pp. 1565-1588, 2011.

[25] M. Hu, J. Chen, Z.-Y. Li et al., "Gold nanostructures: engineering their plasmonic properties for biomedical applications," Chemical Society Reviews, vol. 35, no. 11, pp. 1084-1094, 2006.

[26] B. Ozanne, C. S. Richards, F. Hendler, D. Burns, and B. Gusterson, "Over-expression of the EGF receptor is a hallmark of squamous cell carcinomas," Journal of Pathology, vol. 149, no. 1, pp. 9-14, 1986.

[27] C. R. Leemans, B. J. M. Braakhuis, and R. H. Brakenhoff, “The molecular biology of head and neck cancer," Nature Reviews Cancer, vol. 11, no. 1, pp. 9-22, 2011. 
[28] T. Kobayashi, "Cancer hyperthermia using magnetic nanoparticles," Biotechnology Journal, vol. 6, no. 11, pp. 1342-1347, 2011.

[29] B. Van de Broek, N. Devoogdt, A. Dhollander et al., "Specific cell targeting with nanobody conjugated branched gold nanoparticles for photothermal therapy," ACS Nano, vol. 5, no. 6, pp. 43194328, 2011.

[30] B. Nikoobakht and M. A. El-Sayed, "Preparation and growth mechanism of gold nanorods (NRs) using seed-mediated growth method," Chemistry of Materials, vol. 15, no. 10, pp. 19571962, 2003.

[31] J. Baselga, "The EGFR as a target for anticancer therapy-focus on cetuximab," European Journal of Cancer, vol. 37, no. 4, pp. S16-S22, 2001.

[32] L.-J. Liao, W.-C. Lo, W.-L. Hsu, C.-T. Wang, and M.-S. Lai, "Detection of cervical lymph node metastasis in head and neck cancer patients with clinically N0 neck-a meta-analysis comparing different imaging modalities," BMC Cancer, vol. 12, article 236, 2012. 

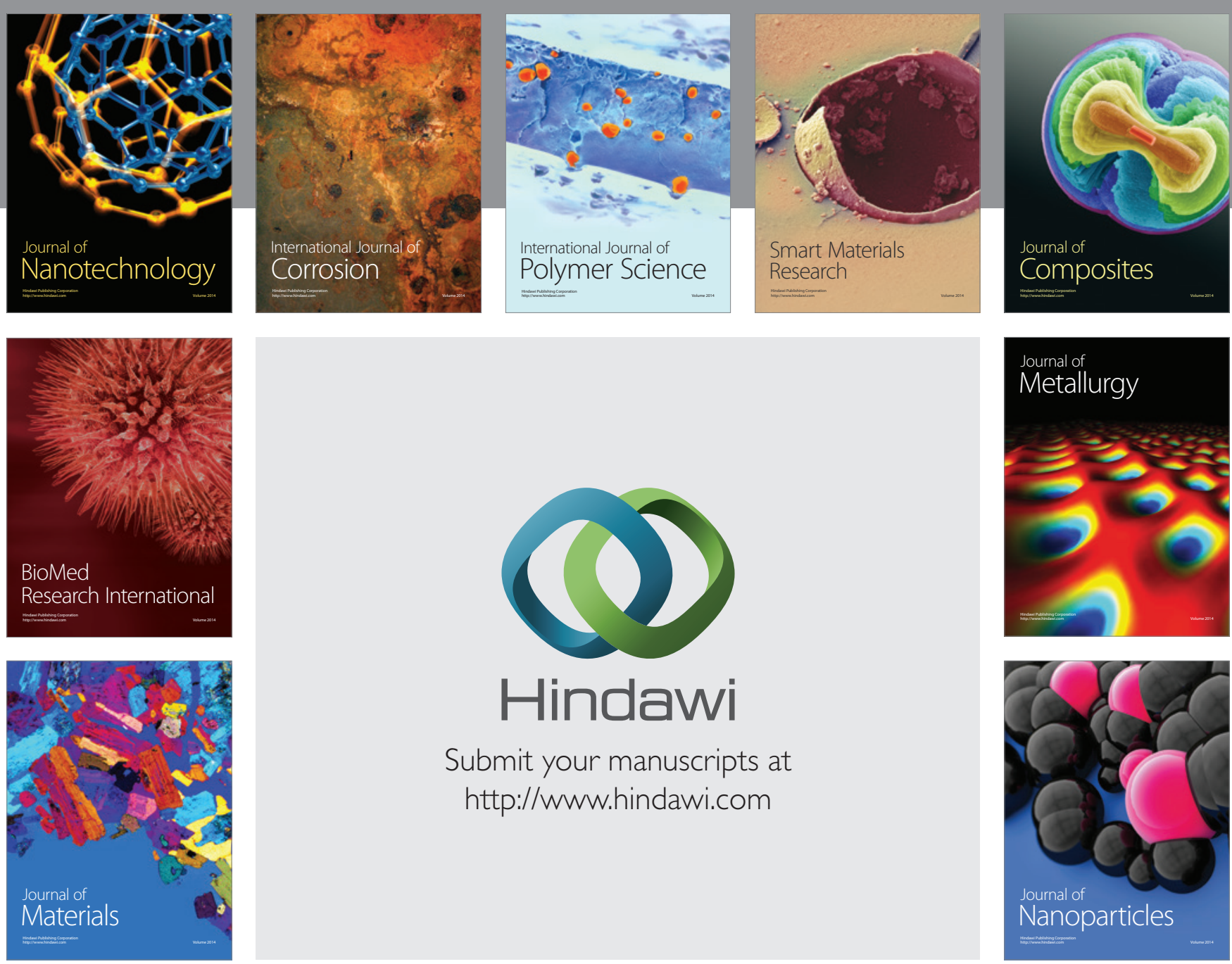

Submit your manuscripts at http://www.hindawi.com
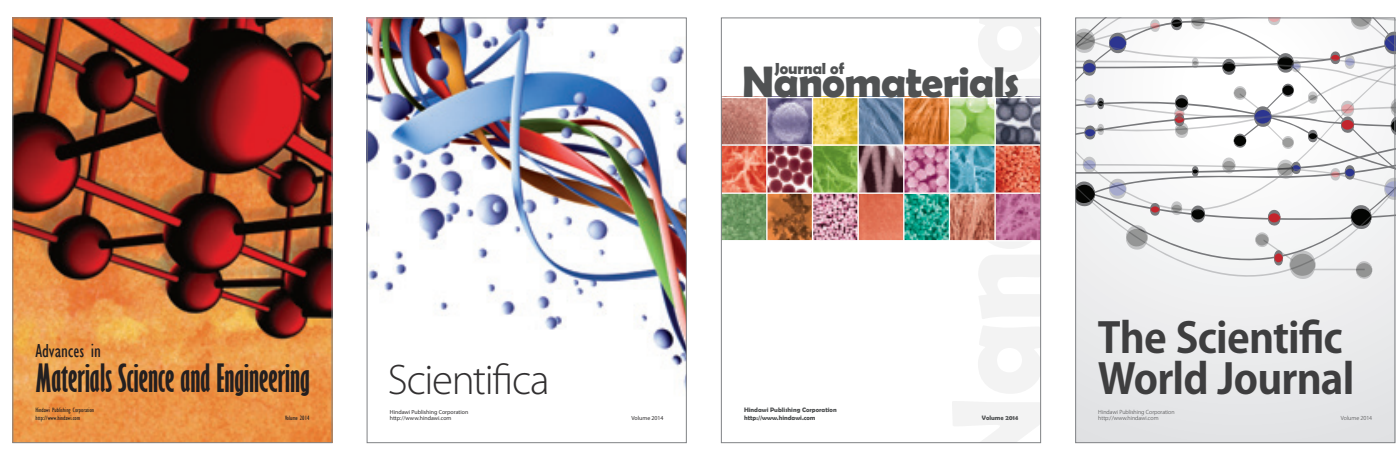

\section{The Scientific World Journal}
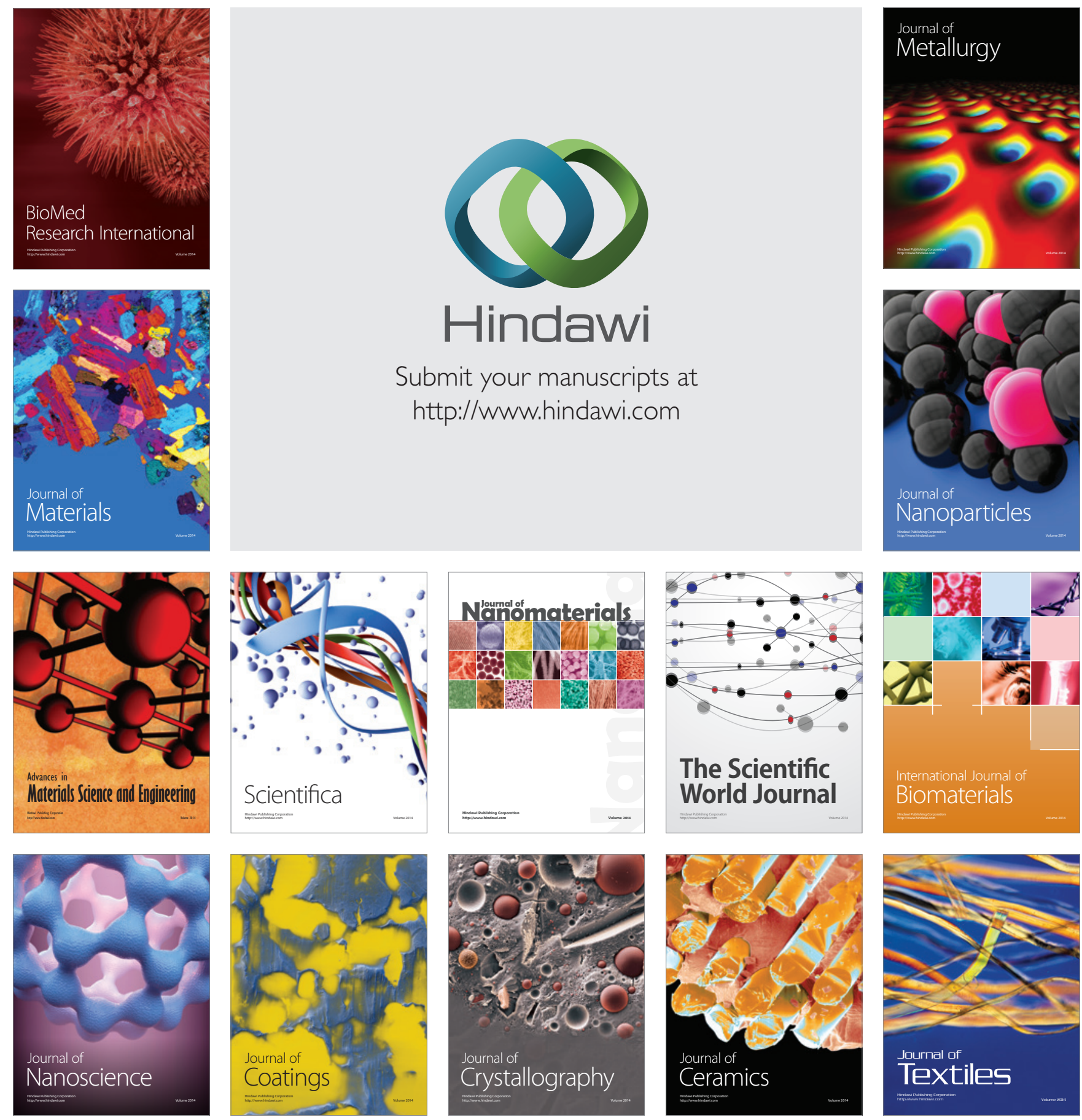